\title{
Análisis multitemporal del cambio de uso del suelo, en el Paisaje Terrestre Protegido Miraflor Moropotente Nicaragua, 1993 - 2011
}

\author{
Veronica Ruiz ${ }^{1}$ \\ Robert Savé2 \\ Alejandrina Herrera Herrera ${ }^{3}$
}

\section{RESUMEN}

Análisis multitemporal del cambio de uso del suelo, en el Paisaje Terrestre Protegido Miraflor Moropotente Nicaragua, 1993 - 2011. El análisis multitemporal permite detectar cambios entre diferentes fechas de referencia, deduciendo la evolución del medio natural o las repercusiones de la acción humana sobre el medio. El propósito del estudio fue evaluar el cambio de uso del suelo en el Paisaje Terrestre Miraflor Moropotente en el período 1993-2011, a través de imágenes satelitales, a fin de determinar el estado de fragmentación del paisaje. Los cambios de usos de suelo fueron derivados de la clasificación de tres imágenes Landsat TM, con una resolución espacial de 30 metros tomadas en febrero de 1993, abril de 2000 y enero 2011. Se realizó una verificación en campo para la identificación de coberturas de suelo y la corroboración en las imágenes satelitales.

La fragmentación se realizó con el cálculo de métricas e índices de fragmentación a nivel del paisaje. Los principales resultados muestran que los cambios de uso de suelo están determinados por la degradación antrópica, principalmente en la conversión de la vegetación nativa a espacios agrícolas y la expansión de la ganadería. El crecimiento demográfico y los monocultivos van ejerciendo presión sobre el bosque, transformando zonas de vocación forestal a cultivos agrícolas. Los cambios de cobertura han significado un paisaje fragmentado con diferentes grados de perturbación, que conllevan a una disminución de la superficie de hábitats naturales, reducción del tamaño de los fragmentos y aislamientos de los mismos.

Palabras claves: paisaje; fragmentación; borde; forma

1 UNAN-Managua/FAREM-Estelí. Estación Experimental para el estudio del Trópico Seco: Correo electrónico: verolisrg@gmail.com 2 Institut de Recerca i Tecnología Agroalimentaries (IRTA), Horticultura Ambiental

3 UNAN-Managua/FAREM-Estelí. Estación Experimental para el estudio del Trópico Seco 


\title{
Multitemporal analysis of land use change in the Terrestrial Protected Landscape Miraflor Moropotente Nicaragua, 1993-2011
}

\author{
Veronica Ruiz ${ }^{1}$ \\ Robert Save ${ }^{2}$ \\ Alejandrina Herrera Herrera ${ }^{3}$
}

\begin{abstract}
Multitemporal analysis to detect changes between different reference dates, deducing the evolution of the natural environment or the impact of human action on the environment. The purpose of the study was to evaluate the change in land use in the Miraflor Moropotente Terrestrial Landscape in the period 1993-2011, through satellite images in order to determine the status of landscape fragmentation. Changes in land use classification were derived from three Landsat TM images with a spatial resolution of 30 meters taken in February 1993, April 2000 and January 2011. We conducted a field verification to identify land cover and corroboration in satellite images.

Fragmentation was performed with the calculation of metrics and indices of landscape-level fragmentation. The main results show that changes in land use are determined by anthropogenic degradation, mainly in the conversion of native vegetation to agricultural areas and the expansion of livestock. Population growth and monocultures are putting pressure on the forest, transformed areas for forestry to agricultural crops. Coverage changes meant a fragmented landscape with varying degrees of disruption, leading to a decrease in surface natural habitats, reducing the size of the fragments and isolates thereof.
\end{abstract}

Keywords: landscape; fragmentation; border; shape.

1 UNAN-Managua/FAREM-Estelí. Experimental Station for the Study of the Dry Tropics E-mail: verolisrg@gmail.com 2 Institut de Recerca i Tecnología Agroalimentaries (IRTA), Environmental Horticulture

3 UNAN-Managua/FAREM-Estelí. Experimental Station for the Study of the Dry Tropics 


\section{INTRODUCCIÓN}

Generalmente los estudios del cambio de uso del suelo forestalevalúan la velocidad de la deforestación/ reforestación, determinan su extensión geográfica y entienden cuáles son las causas sociales y económicas de los cambios, en las escalas global, regional y local (Skole et al. 1994; Ojima, et al. 1994). El análisis multitemporal permite detectar cambios entre diferentes fechas de referencia, deduciendo la evolución del medio natural o las repercusiones de la acción humana sobre ese medio (Chuvieco 1996).

Los ecosistemas terrestres han sufrido grandes transformaciones, la mayoría debido a la conversión de la cobertura del terreno y a la degradación e intensificación del uso del suelo (Lambin 1997). La actividad humana ha sido la gran transformadora del paisaje en los últimos 300 años (Aguayo et al.2009). Por ejemplo los bosques y selvas de América Latina para el año 2000 se habían reducido en más de un 50 $\%$ de su cobertura original; en particular, países como Brasil, México y Costa Rica fueron el centro de las mayores alteraciones (Velázquez et al. 1997).

En Nicaragua la vegetación ha sufrido perdida de los bosques producto de la colonización agrícola, el aprovechamiento de los bosques sin control y la expansión de la ganadería extensiva (Stevens 2001). Otros factores del cambio de usos de suelos es: migraciones campesinas, el reasentamiento de los desmovilizados de guerra y orientación de las políticas y programas de desarrollo agropecuarias (INAFOR 2008). Los bosques actuales se encuentran en las áreas protegidas (tienen por objeto la conservación, el manejo racional, la restauración de la flora, fauna silvestre, la biodiversidad, la biosfera, fenómenos geomorfológicos, sitios de importancia históricos, arqueológica y cultural), sin embargo se están modificando a pesar que existen categorías de manejoen los que incluyen directrices para la administración. La categoría de manejo es una denominación técnica que se da a un área protegida en función de la valoración de las características biofísicas y socioeconómicas intrínsecas del área y los objetivos de conservación que puede cumplir. Cada una representa diversos grados de intervención humana y tiene sus propias restricciones en cuanto al uso de los recursos.

En los últimos años se han realizados estudios sobre cambio de uso de suelo y escenarios de cambio climático (Ticona 2011),evaluación de áreas afectadas, tras el paso del huracán Félix en el año 2007 (Mendoza 2010), análisis multitemporal para la detección de cambios en el uso del suelo en tres municipios afectados por el huracán Juana (Williamson 2006), evaluación de un paisaje fragmentado para la conservación y recuperación de la biodiversidad en el Paisaje Terrestre Protegido Miraflor Moropotente (PTP MM) Estelí, Nicaragua (Correa 2000).

El análisis del cambio de uso de suelo en el PTP MM puede utilizarse para reorientar los programas de manejo y la restauración de ecosistemas degradados del área protegida. Por otra parte los resultados pueden mostrar el grado de fragmentación del área, referidos por atributos (número de fragmentos, tamaño, forma, abundancia, riqueza y grado de aislamiento de los fragmentos). El presente trabajo tiene como objetivo evaluar el cambio de uso del suelo y fragmentación en el Paisaje Terrestre Miraflor Moropotente en el período 1993- través de imágenes satelitales.

\section{MATERIALES Y MÉTODOS}

Descripción del área de estudio. El estudio se ubica en el PTP MM, en la Región Central Norte de Nicaragua, entre las coordenadas $13^{\circ} 29^{\prime} 50^{\prime \prime}$ Latitud Oeste a $25^{\circ}$ 29'15" y $86^{\circ} 7$ '30" longitud Norte a 86 3' 22'. A una distancia de la ciudad de Estelí (Fig.1).

La precipitación promedio anual varía entre $800 \mathrm{y}$ en las zonas más secas (bosque decido y mesas de Moropotente) y entre 1200 y 1600 en las zonas más 
húmedas (bosque mixto y montano). La temperatura promedio anual varía entre 22 y en las zonas más bajas y menos de en las zonas más altas (Correa 2000).

El $57 \%$ del área es de vocación forestal, tierras que, debido a sus características físicas del suelo, topografía y pluviosidad, deberían ser mantenidas bajo cobertura boscosa u otra utilización sostenible que pueda evitar externalidades negativas relacionadas al suelo y agua. (INAFOR 2008), el $11.7 \%$ pecuaria, el 11.8 $\%$ agrícola con cultivos perennes, el $7.3 \%$ cultivos especiales (helechos y orquídeas) y el $12.2 \%$ con cultivos anuales En relación al paisaje, se diferencian cuatro tipos: bosque deciduo, bosque montano, mesas de moropotente y bosque mixto (Correa 2000).

En el año 2004 dado a su extensión territorial y otros valores intrínsecos, es declarada como Paisaje Terrestre Miraflor Moropotente. Su categoría ha venido cambiando, pasando en el caso de Moropotente por Reserva natural en 1991 y como Reserva Natural

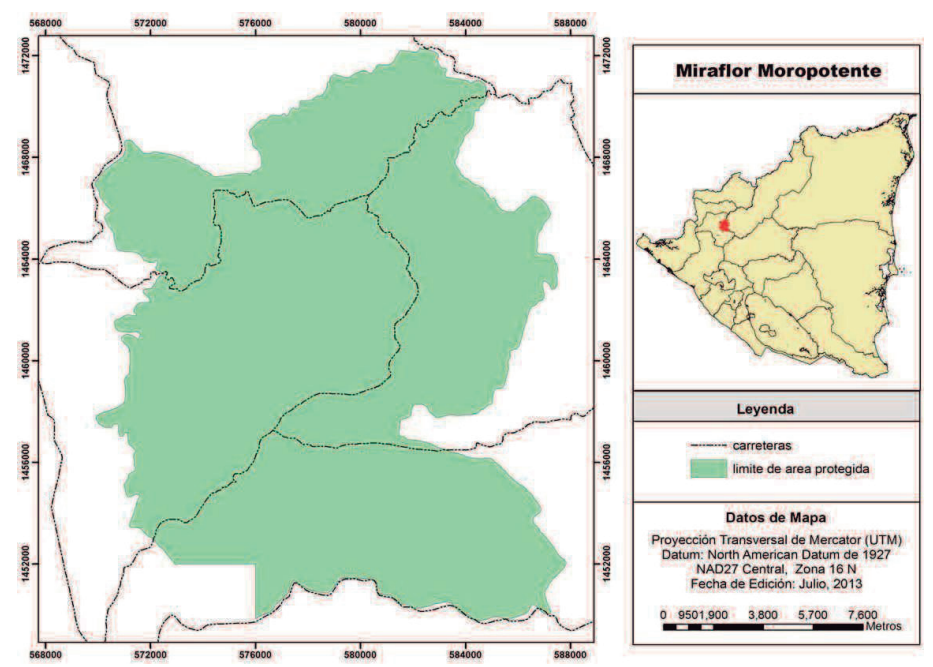

Figura 1. Ubicación geográfica del Paisaje Terrestre Protegido Miraflor Mo- ropotente. En la imagen de la izquierda mapa de

Nicaragua, figura de la de- recha PTP MM.

de Miraflores en 1996. Por otra parte estas dos áreas conforman la Reserva Natural Miraflor Moropotente, declarada como área demostrativa en el año 1999. Actualmente el área es manejada por el FORO
Miraflorque aglutina a las diferentes organizaciones del área protegida y tiene como función principal la gestión y promoción del manejo y desarrollo sostenible del área protegida, las directrices de administración están dirigidas a: controlar, normar la investigación y monitoreo en el área, permitir actividades económicas, restauración de paisajes y prohibir la introducción de especies exóticas (Nicaragua 1999).

\section{Determinación de los cambios de uso del suelo a} través de imágenes satelitales. Para la estimación, el análisis y la cuantificación de los cambios de uso del suelo a través del tiempo, se utilizaron tres imágenes Landsat TM, con resolución espacial de 30 metros tomadas en los meses febrero de 1993, abril de 2000 y enero 2011, en una serie temporal de 18 años (19932011). Las imágenes fueron obtenidas desde USGS (centro Geológico de los Estados Unidos), cuyo nivel de procesamiento incluye correcciones radiométricas, geométricas y topográficas.

Clasificación de las imágenes. Se seleccionaron áreas representativas de cada cobertura del suelo para obtener su valor espectral, clasificando píxeles que representaron los patrones de los tipos de cobertura del suelo y que se identificaron en campo. Este tipo de clasificación en donde se indica a priori cuáles serán las clases temáticas, se denomina supervisada (ERDAS, Inc. 1997).

Los pasos que se siguieron fueron: (1) selección y edición: identificación de pixeles similares y representativos de las áreas de entrenamiento asignándoles a una firma espectral determinada y calculándole su estadístico con apoyo en los trabajos de campo. (2) evaluación de las categorías o firmas espectrales; una vez definidas las firmas espectrales y antes de abordar el proceso de clasificación, se evaluó la viabilidad de las categorías para su clasificación. (3) Clasificación de la imagen satelital: el algoritmo de la clasificación supervisada empleado es del tipo paramétrico. Se trabajó al $95 \%$ de confiabilidad de la 
clasificación de la imagen correspondiente al año 2011, con un error de $\pm 5 \%$. El software utilizado para la clasificación de imágenes ERDAS 2011 y ArcGIS 10.

Cobertura del suelo. La determinación del tipo de cobertura vegetal del área, se basó en la clasificación de uso de suelo del Ministerio Agropecuario y Forestal (Nicaragua 2010): (1) bosque latifoliado cerrado (altos o bajos, con cobertura de copas $>70$, (2) bosque mixto: pino y roble, (mezcla de especies de diferentes gradientes altitudinales, por ejemplo: 50 $\%$ roble, y en algunos casos $50 \%$ pino), (3) bosque latifoliado abierto (altos o bajos, con cobertura entre $30-70 \%$ ), (4) tacotal (vegetación leñosa derivada de la tala del bosque, presenta un mosaico de varias fases de sucesión, frutales con árboles),(5) pastos con árboles dispersos (sistemas agroforestales, naturales o sembrados), (6) pastizales (pastos mejorados, pastos con malezas y/o árboles esparcidos), (5) cultivos agrícolas (áreas que están dedicadas a todo tipo cultivos anuales tecnificados o no).

Verificación en campo de las clases de cobertura, diseño y tamaño del muestreo. Se realizó una verificación en campo para la identificación de coberturas de suelo y la corroboración en las imágenes satelitales. En la imagen del 2011 se trazó una red de puntos al azar. Según (Congalton 1988), los puntos de verificación tienen que representaruna superficie aproximada a $1 \%$ de la superficie cartografiada. En el área de estudio representó155 puntos del área total.

Análisis y cuantificación de los cambios. El cambio de uso de suelo fue cuantificado usando la ecuación propuesta por (FAO 1996), para calcular la tasa de cambio anual

$$
\text { (TDA): } \quad \text { Tasa }=\left[\frac{S_{2}}{S_{1}}\right]^{1 / n}-1
$$

Dónde: TDA: tasa de cambio anual: $\mathrm{S} 2=$ superficie fecha $2, \mathrm{~S} 1$ = superficie en la fecha $1, \mathrm{n}=$ es el número de años entre las dos fechas

Fragmentación del PTP MM. A partir del uso actual de suelo realizado con teledetección (imágenes de satélite Landsat 7 del año 2011), se realizó el cálculo de métricas e índices de fragmentación a nivel de Paisaje. El procedimiento de cálculo se aplicó mediante las herramientas SIG V LATE y PATCH ANALYST ambas diseña- das para el software ArcGISversión 9.3 y los índices se calcularon con la herramienta V LATE.

Métrica de área. Corresponde a la suma total de todas las áreas (m2) de un tipo de clase de vegetación, expresado en hectá- reas (McGarigal et al 2002).Se calculó mediante la fórmula:

$\mathrm{n}$

$$
\begin{aligned}
& \mathrm{CA}=\Sigma \mathrm{a}_{\mathrm{ij}}(1 / 10.000) \\
& \mathrm{j}=1
\end{aligned}
$$

Dónde: CA: área total y aij:área de cada parche correspondiente a cada clase $(\mathrm{m} 2)$

Métrica de borde. Se obtiene a partir de la sumatoria de la lon- gitud (en metros) de cada uno de los segmentos que componen los parches de una clase o un tipo de cobertura, dividido entre el área total del paisaje (m2) y expresado en hectáreas. Usando la siguiente fórmula:

$$
E D=\frac{\sum_{k=1}^{m} e_{i k}}{A(10.000)}
$$

Donde: ED: Indice de Borde. eik: longitud de cada segmento que compone los parches de cada una de las clases (metros) y A: área total del paisaje (m2)

Métrica de forma. Para cuantificar esta relación se utilizaron dos índices estándar: índice de diversidad de forma propuesto por (Patton 1975) adaptado por (Laurence y Yensen 1991) 


$$
S H A P E=\frac{P_{i j}}{\min P_{i j}}
$$

Donde $\mathrm{Pij}=$ perímetro de cada parche $(\mathrm{m})$ y $\min \mathrm{Pij}=$ perímetro máximo posible de cada parche relativamente compacto.

Este índice toma valores de 1 o cercanos a 1, mientras más compacto sea el parche y este asciende hasta el infinito mientras más irregular o menos compacto sea el fragmento. Los resultados de este índice pueden ser agrupados en cinco rangos (Henao 1988): redondo (SI $<1.25$ ), oval redondo (SI entre 1.25 a 1.5), oval oblongo (SI entre 1.5 a 1.7), rectangular oblongo (SI 1.7 a 2 ) y amorfo (SI $>2)$.

Índice de dimensión fractal promedio de un fragmento discreto (Krummel et al 1987),

$$
F R A C=2 \ln \left(0.25 P_{i j}\right) \ln A_{i j}
$$

Dónde: Pij: Perímetro del parche (m) y Aij: Área de cada parche (m2).

Este índice varía entre 1 para formas euclidianas simples (cua- drados y círculos) y 1.001 a 2 para formas complejas análogas a objetos fractales. La forma de los parches será más irregular.

Análisis estadísticos. El análisis se basó en la estadística des- criptiva, reflejada en medias, porcentajes desviación y errores es- tándares. Se utilizó análisis descriptivo multivariado exploratorio para determinar si la muestra proviene de una distribución normal y el análisis de componentes principales y conglomerados (Di Rienzo et al. 2010). Los datos que no cumplen con el supuesto fue- ron normalizados con $\log 10$.

\section{RESULTADOS}

\section{Cambio de uso del suelo período 1993-2001}

Para los períodos evaluados, los resultados evidencian cambios de cobertura para el área protegida. En el primer período (1993- 2000) el bosque latifoliado denso refleja un ligero incremento, sin embargo, para el siguiente período (2000-2011) disminuye. El bos- que mixto (pino y roble) en ambos períodos ha sufrido una dismi- nución. El bosque latifoliado ralo en el primer período evaluado ha disminuido, sin embargo, aumenta para el siguiente período. Todo esto se refleja en los tres mapas generados. La superficie dedicada a pastos con árboles dispersos aumenta en el primer período $\mathrm{y}$ en el siguiente experimenta una disminución. Los pastos disminuyen en ambos períodos, sin embargo, los tacotales y áreas de cultivos aumentan. (Figs. 2, 3 y 4).

Las tasas de cambio (TAC) en el periodo evaluado fueron de 28.17 hectáreas en aumento y 22.42 hectáreas en disminución (Tabla 1).

\section{Fragmentación del paisaje período 1993-2001}

Los resultados indican un paisaje fragmentado que conlleva a una disminución de la superficie de hábitats naturales (Fig. 5). Al aplicar el índice de diversidad de forma de Patton, el bosque latifo- liado denso muestra formas amorfas o irregulares en relación al resto de coberturas que tienen formas más definidas (rectangulares u oblongas). Mientras que el índice de dimensión fractal refleja que todas las categorías presentan formas irregulares. La métrica que posee mayor borde promedio son las áreas de bosque latifoliado denso, seguido de las áreas de pastos con árboles dispersos. Las áreas que presentan menores bordes son los cultivos y las áreas de bosque mixtos. Se refleja que cuando los bordes son muy abrup- tos, se evidencia más el cambio de uso del suelo (Tabla 2). Los ta- cotales ocupan la mayor superficie del área total (26.12\%), los bosques ralos el $23.89 \%$, pastos con árboles dispersos el $21.46 \%$, bosque denso con $14.19 \%$, pastizales $8.25 \%$, cultivos agrícolas $5.38 \%$ y bosque mixto $0.71 \%$. Los tacotales presentan la mayor cantidad de parches, seguidamente bosques ralos y pastos con ár- boles dispersos. Los cultivos, pastizales, bosque denso y mixto, menor número de fragmentos (Fig. 6). 


\section{DISCUSIÓN}

\section{Cambio de uso del suelo período 1993-2001}

El paisaje del área protegida PTPMM presenta todavía rema- nentes de bosque nativo, pero es un paisaje fragmentado por la ac- tividad agrícola y pastoril. (Correa 2000). Este cambio de uso del suelo inicia con el auge de la ganadería y el boom del café en los años 70' (Ravera 2007). Posteriormente, en los años 80' con la Re- forma Agraria, se introduce el cultivo de la papa (Solanum tubero- sum) como principal alternativa económica haciendo mayor presión por el bosque nativo (López 2002). La dinámica de cambio es desencadenada y controlada por factores demográficos y las conse- cuentes actividades productivas Para el área, según (PANIF 1999), desde el año 1999 al 2005 (6 años), la población ha incrementado en un $53 \%$. De 7,500 habitantes pasaron a 16,127 (INIDE 2005).
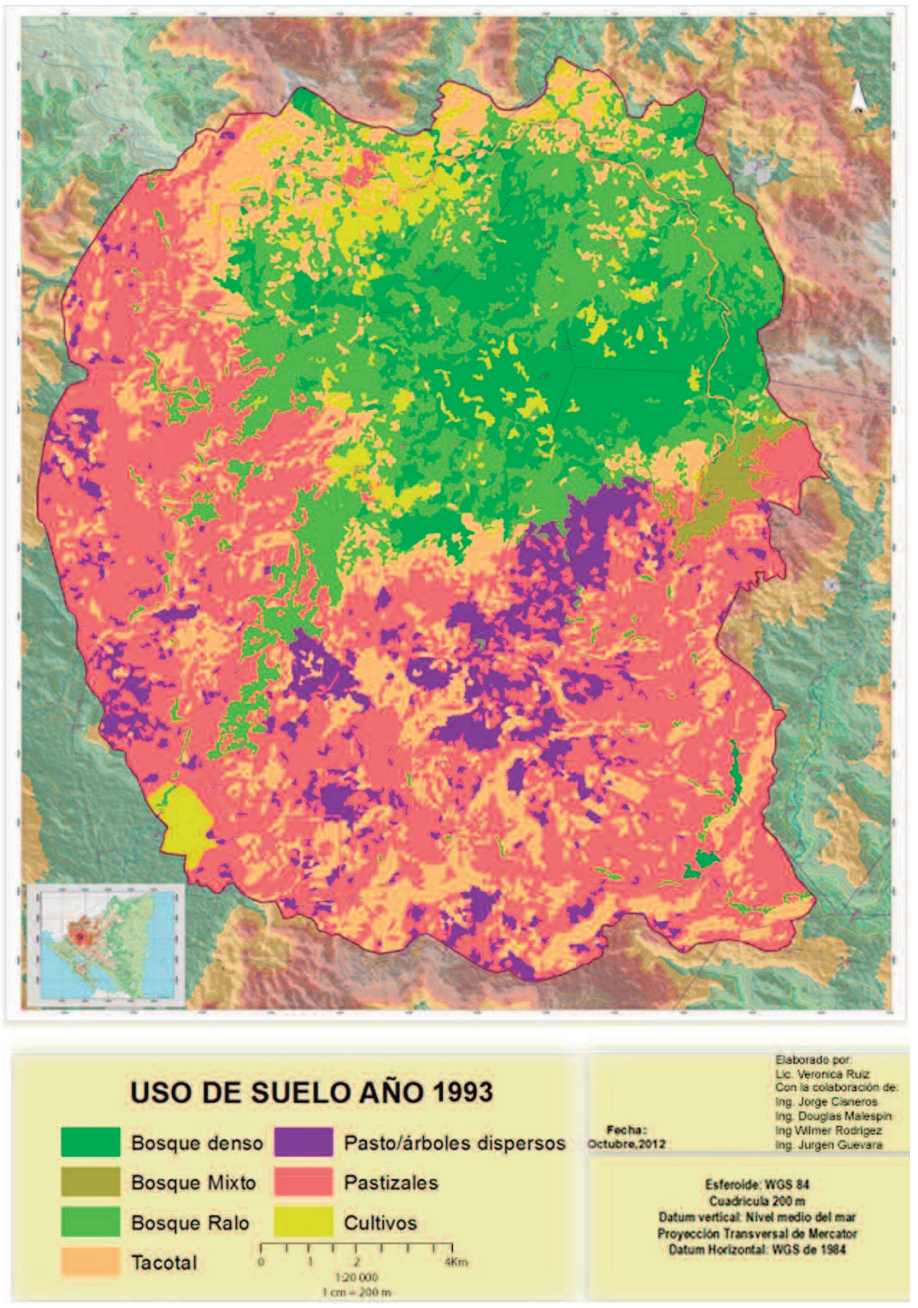

Figura 2. Mapa de cambios de uso para el año 1993
El ligero aumento del bosque latifoliado denso en el primer perí- odo de evaluación (1993-2000) puede obedecer a que en este perí- odo toma impulso una reflexión entre la municipalidad de Estelí y la Unión de Cooperativas Agropecuarias de la zona, sobre las caracte- rísticas y el desarrollo del área. Se parte de la premisa de que era una zona con alto potencial ecológico y productivo, pero muy afec- tado por la forma de uso a la que se le sometió (MARENA et al 2004).

Desde ese entonces, se promueve la declaratoria de Miraflor como Área Protegida, la cual se concretiza en el año 1999 junto a todos los cerros y macizos del país como una Reserva Natural. Sin embargo, a partir del 2000 al el 2011, se evidencia una disminución del bosque latifoliado denso, que se puede deber a la presión ejer- cida por el aumento de la población en el área para este período (INIDE 2005).
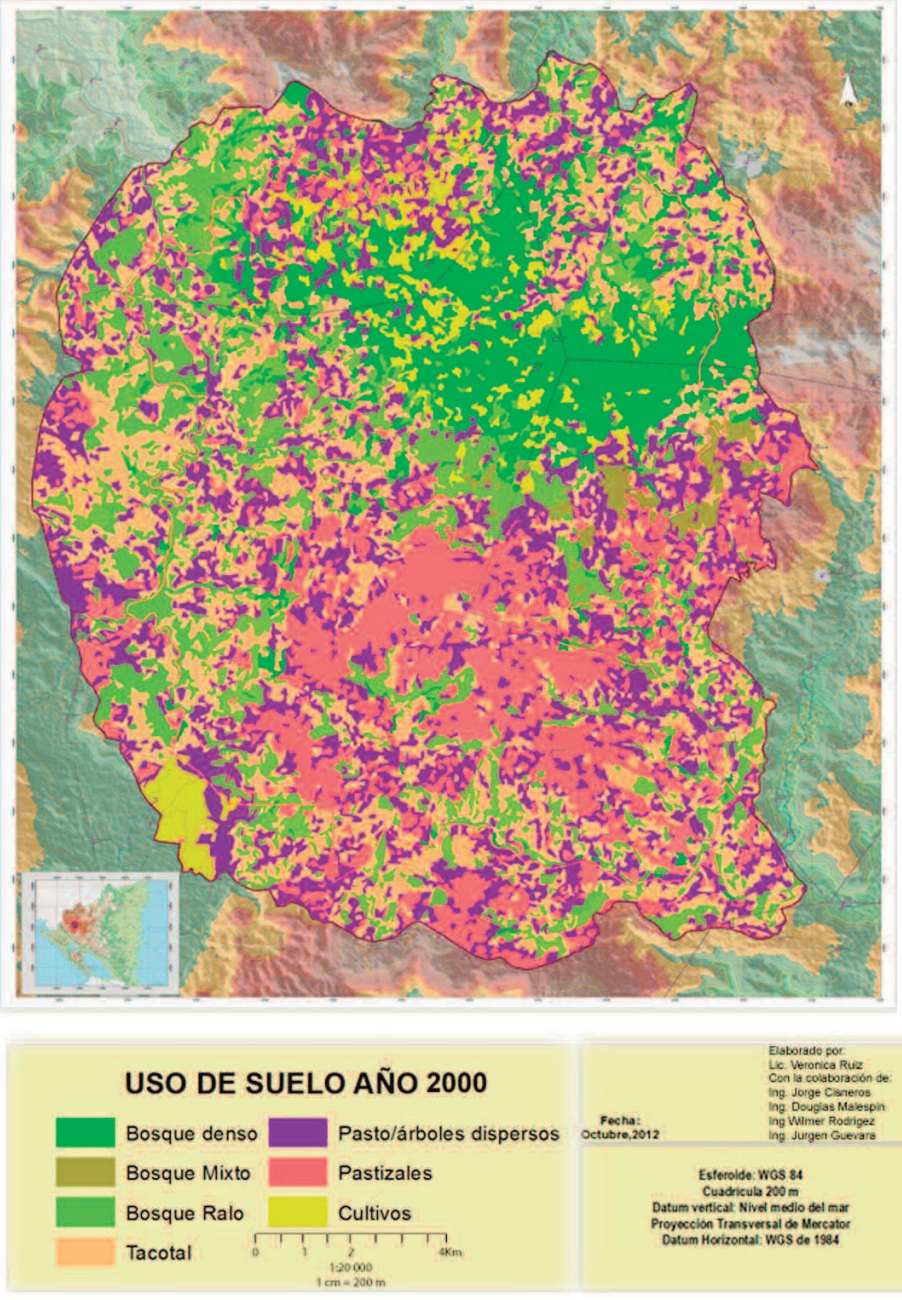

Figura 3. Mapa de cambios de uso para el año 2000 

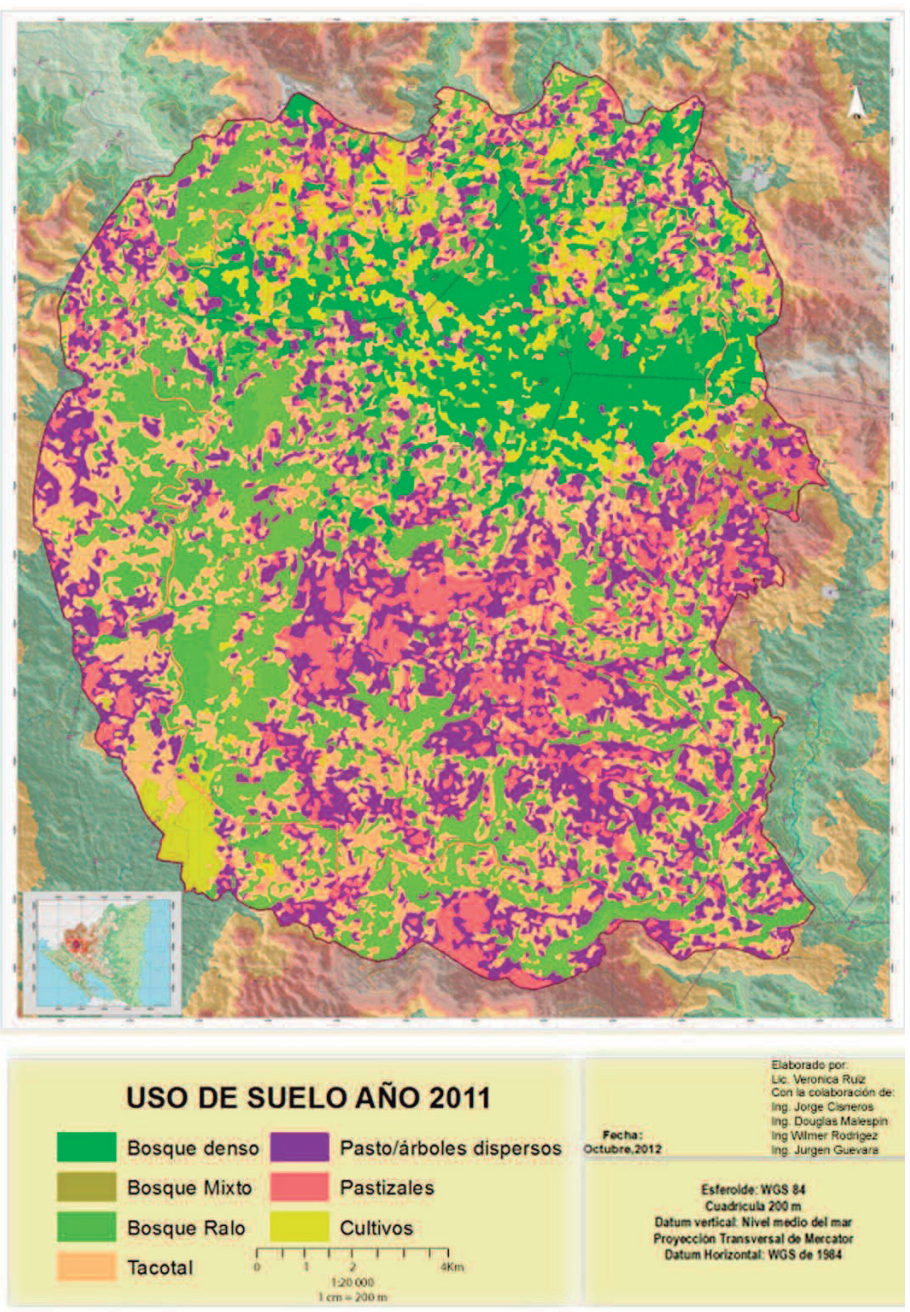

Figura 4. Mapa de cambios de uso para el año 2011

Tabla 1. Cobertura del suelo y tasa de cambio (TAC) en el período $1993-2011$.

\begin{tabular}{|c|c|c|c|c|c|c|c|c|}
\hline \multirow{2}{*}{ Cobertura } & \multicolumn{2}{|c|}{ Áreas de 1993} & \multicolumn{2}{|c|}{ Áreas de 2000} & \multirow{2}{*}{$\begin{array}{l}\text { TAC } \\
\mathrm{Ha} \\
\end{array}$} & \multicolumn{2}{|c|}{ Áreas de 2011} & \multirow{2}{*}{$\begin{array}{l}\text { TAC } \\
\text { Ha }\end{array}$} \\
\hline & Ha & $\%$ & Ha & $\%$ & & Ha & $\%$ & \\
\hline $\mathrm{BLD}^{1}$ & 5226.6 & 11.11 & 6101.2 & 13.0 & 2.2 & 5520.4 & 11.8 & -0.91 \\
\hline DIVI & 472.4 & 1.01 & 462.6 & 0.9 & -0.3 & 306.4 & 0.6 & -3.67 \\
\hline $\mathrm{BLR}^{3}$ & 8971.6 & 19.17 & 8625.5 & 18.4 & -0.5 & 11867.9 & 25.3 & 2.94 \\
\hline $\mathrm{PAD}^{4}$ & 3689.1 & 7.88 & 10398.0 & 22.2 & 15.9 & 10155.0 & 21.7 & -0.21 \\
\hline $\mathrm{Pas}^{5}$ & 17340.4 & 37.06 & 7497.4 & 16.0 & -11.2 & 3963.4 & 8.4 & -5.63 \\
\hline $\mathrm{Tac}^{6}$ & 9162.2 & 19.58 & 11673.1 & 24.9 & 3.5 & 12329.2 & 26.3 & 0.50 \\
\hline $\mathrm{Cul}^{7}$ & 1932.4 & 4.13 & 2036.9 & 4.3 & 0.7 & 2652.3 & 5.6 & 2.43 \\
\hline Total & 46794.9 & 100 & 46795.0 & 100 & & 46795.0 & 100 & \\
\hline
\end{tabular}

1 BLD: Bosque latifoliado denso, 2 BM: Bosque Mixto, 3 BLR: Bosque latifo- liado ralo, 4 PAD: Pasto/árboles dispersos, 5 P: Pastizales, 6 T: Tacotal, 7 C: Cultivo.

Valores por debajo de cero, corresponden a categorías que están perdiendo superficie y los de arriba de cero, los que están ganando.

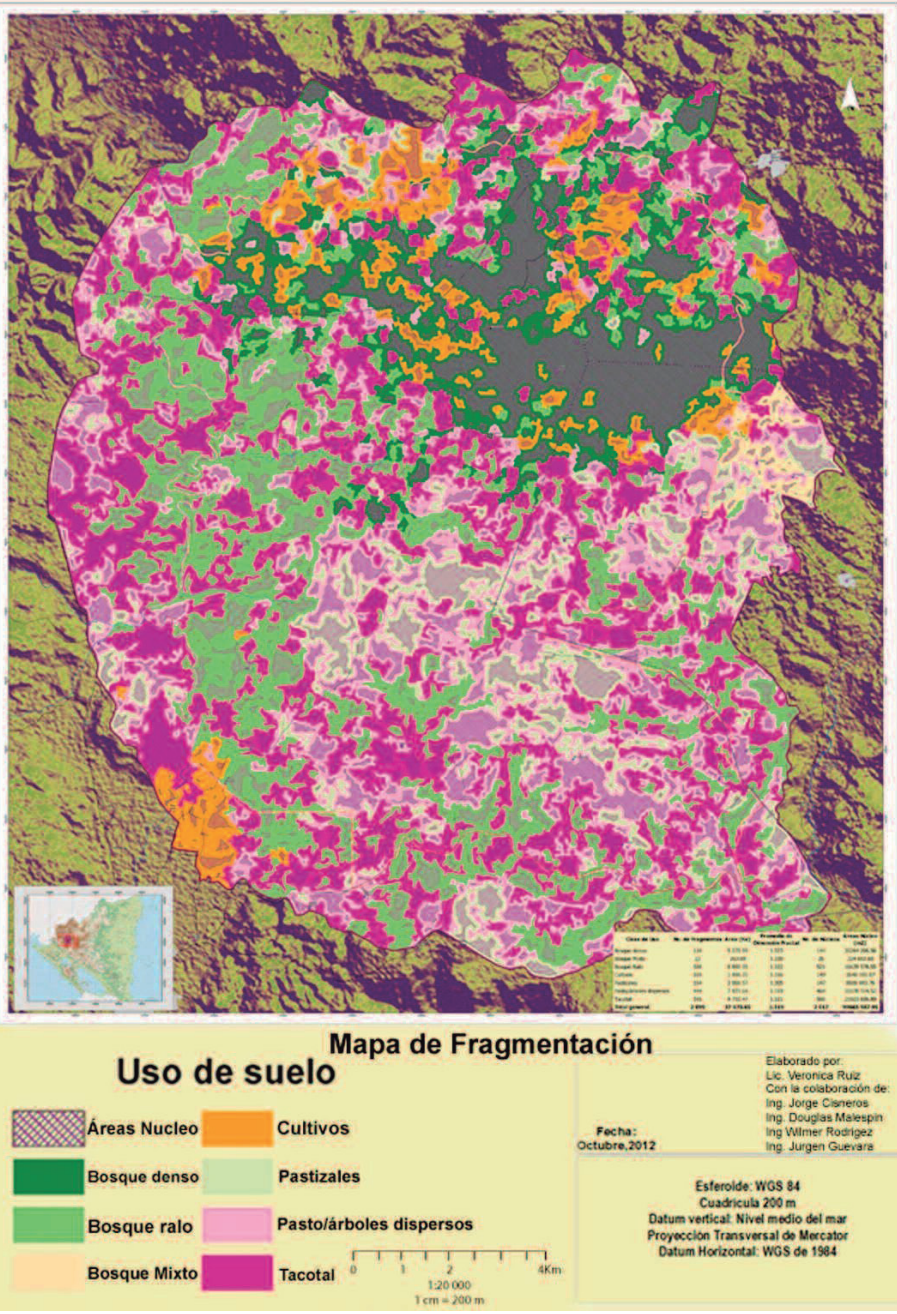

Figura 5. Fragmentación por cada tipo de cobertura en el PTP MM Terrestre Miraflor Moropotente en el 2011.

Probablemente la extracción de productos maderables y la expansión de la ganadería en la zona, ha hecho presión sobre el bosque mixto (pino y roble), provocado su disminución en ambos períodos evaluados. La pérdida de coberturas forestales densas ha dado paso a un incremento en otros usos y en cubiertas fo- restales de menor densidad (Maas et al 2005). El bosque latifoliado ralo ha aumentado en relación al primer período, lo que puede estar asociado a diversas acciones que se están desarro- llando vinculadas al manejo sostenible del área y contempladas en el plan de manejo, con el fin de lograr la conservación de los recursos naturales y la sostenibilidad productiva (MARENA et al 2004).

Diversos programas han promovido incentivos para la incorpo- ración de sistemas silvopastoriles en las 
fincas de los productores del área, esto puede verse reflejado en el aumento en el área de pastizales con árboles dispersos (pastizales arbolados) en relación al primer período de evaluación.

Las áreas de pastizales se encuentran en un avanzado estado de deterioro, los bajos costos de los subproductos de la ganadería, las fluctuaciones en los precios y los altos costos de inversión para esta actividad, puede estar provocando la disminución del área para esta práctica. Por consiguiente los potreros se abandonan por los altos costos de mantenimiento, dado lugar a áreas con tacotales en donde reaparecen otras especies invasoras y colonizadoras (Ravera 2007).

Sin embargo, la práctica agrícola sigue expandiéndose y se re- fleja en un aumento en área en los últimos años evaluados. A pesar de que es un área protegida, actualmente tiene una categoría más flexible en términos de uso, permitiendo cierto grado de intervención. Probablemente este es uno de los factores que influye en el aumento de áreas dedicadas a la agricultura y la consecuente dis- minución de las áreas boscosas.

Tabla 2. Índice de cobertura de forma y borde.

\begin{tabular}{llcc}
\hline \multirow{2}{*}{ Cobertura } & $\begin{array}{c}\text { Índice de diversidad } \\
\text { Patton (forma) }\end{array}$ & $\begin{array}{c}\text { Índice de dimensión } \\
\text { Fractal (forma) }\end{array}$ & Índice de borde \\
\cline { 2 - 4 } & \multicolumn{1}{c}{$\sigma \pm \mathrm{SE}$} & $\sigma \pm \mathrm{SE}$ & $\sigma \pm \mathrm{SE}$ \\
$\mathrm{BLD}^{1}$ & $2.03 \pm 0.08$ & $1.32 \pm 0.003$ & $4784.17 \pm 2.35$ \\
$\mathrm{BM}^{2}$ & $1.81 \pm 0.06$ & $1.32 \pm 0.005$ & $2169.53 \pm 1.02$ \\
$\mathrm{BLR}^{3}$ & $1.87 \pm 0.02$ & $1.32 \pm 0.001$ & $2551.10 \pm 1.22$ \\
$\mathrm{PAD}^{4}$ & $1.90 \pm 0.03$ & $1.31 \pm 0.002$ & $2911.81 \pm 1.53$ \\
$\mathrm{Pass}^{5}$ & $1.70 \pm 0.03$ & $1.30 \pm 0.002$ & $2499.00 \pm 1.03$ \\
$\mathrm{Tac}^{6}$ & $1.88 \pm 0.02$ & $1.32 \pm 0.001$ & $2713.97 \pm 1.42$ \\
$\mathrm{Cul}^{7}$ & $1.76 \pm 0.02$ & $1.31 \pm 0.002$ & $2123.06 \pm 1.01$ \\
\hline
\end{tabular}

La $\sigma$ es la media y la SE el error estándar 1 BLD: Bosque latifoliado denso. 2 BM: Bosque Mixto. 3 BLR: Bosque latifoliado Ralo. 4 PAD: Pasto/árboles dispersos. 5 P: Pastizales. 6 T: Tacotal. 7 C: Cultivos.

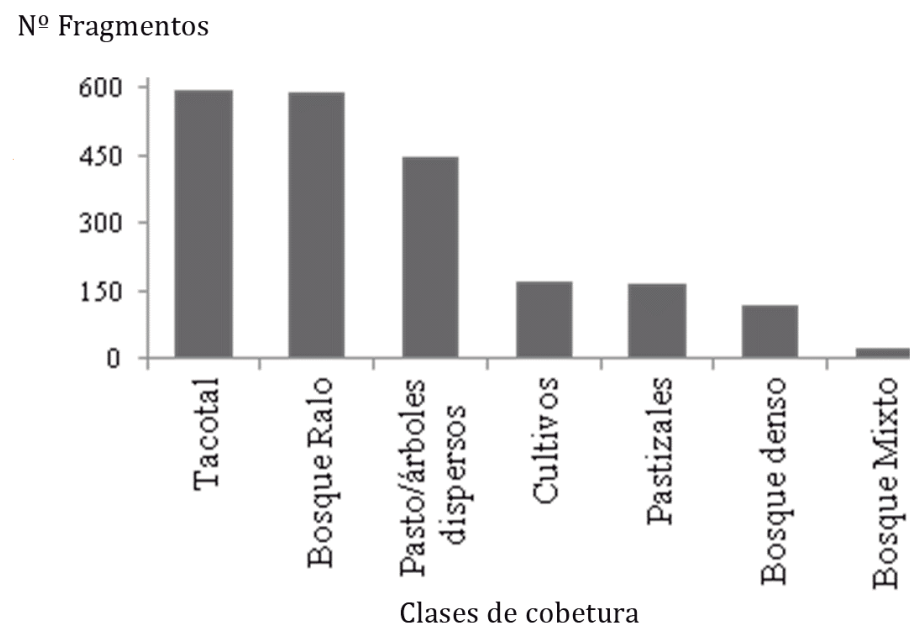

Figura 6. Fragmentos por cada tipo de cobertura en el PTP MM.

\section{Fragmentación del paisaje}

El estado de conservación del recurso bosques del PTP MM presenta fragmentos de hábitat natural bajo una fuerte presión, como producto de factores antropogénicos (MARENA et al 2004). La fragmentación del bosque nativo se da por el reemplazo de grandes áreas por otros ecosistemas, dejando parches (o islas) se- parados del bosque y entre sí (Murcia 1995). En el área, se evi- dencia en los diferentes tamaños y formas de los fragmentos que reflejan las diferentes coberturas paisajísticas.

El grado de intervención del área da lugar a una fragmentación excesiva de los ecosistemas y puede reducir la aptitud de un hábitat para ciertas especies, al no existir fragmentos suficientemente gran- des para mantener poblaciones estables (Usher 1987). Se sabe que las modificaciones humanas son las más determinantes en la frag-mentación de los ecosistemas, acelerando la perdida de hábitat y la reducción de la diversidad biológica. El paso de bosque nativo a áreas de pastizales y agrícolas en el área, hace que el paisaje se encuentre más fragmentado y el efecto de borde sea más abrupto.

La forma de los parches es de importancia ecológica, especial- mente por afectar directamente los 
movimientos y flujos entre eco- sistemas adyacentes (Forman 1995, citado por Correa 2000). Para el área, la formas irregulares de los fragmentos en el paisaje boque denso y ralo, probablemente se deben a patrones históricos de fragmentación (Correa 2000).

Las formas regulares en el paisaje de tacotales, pastos con ár- boles dispersos, pastizales y cultivos pueden estar relacionadas con la intervención del ser humano, en la delimitación de sus terri- torios, Correa, 2000). El índice fractal indica que todos los fragmen- tos se asemejan a formas complejas (Krummel et al. 1987; Rau et al. 2000; Rau y Gantz 2001), dando lugar a diversidad de paisajes.

La fragmentación genera cambios en los procesos ecológicos y uno de esos cambios es la presencia de los efectos de los bordes, como resultado de la interacción de dos ecosistemas adyacentes, (Murcia 1995). El efecto borde será un factor a tener en cuenta los cambios y/o alteraciones en la biodiversidad de los ecosistema (Essen et al. 2006).

\section{CONCLUSIONES}

Se estudió el cambio de uso de suelo en el Paisaje Terrestre Protegido Miraflor Moropotente en una serie de 18 años haciendo uso de imágenes satelitales Landsat en dos períodos históricos (1993-2011). Se evidencia cambio de uso del suelo mostrado en diferentes paisajes.

El aumento de la población a partir de los años 80', las prácticas agrícolas y pecuarias hacen presión sobre el bosque latifoliado denso nativo y el bosque mixto provocando una fragmentación del mismo. Aunque existe un documento rector para el área protegida, los escasos recursos humanos para el control, la flexibilidad en la categoría de manejo ha permitido la práctica de la agricultura y la ganadería con la consecuente fragmentación del bosque.
El paisaje más dominante son los tacotales, que corresponde a áreas agrícolas y de pastizales abandonados dando lugar a la re- generación de especies nativas y la colonización de pioneras.

Determinar el cambio de uso del suelo en esta área de impor- tancia nacional para la conservación de la biodiversidad y de re- carga hídrica, de igual manera para la planificación, ordenamiento del territorio y ejecución de acciones de restauración de los ecosistemas fragmentados.

\section{AGRADECIMIENTOS}

A Fundación Autónoma Solidaria. Universidad Autónoma de Barcelona, al Proyecto Integral de Manejo de Cuencas Hidrográfi- cas, Agua y Saneamiento (MARENA-PIMCHAS) por su apoyo fi- nanciero. Este trabajo está integrado en el proyecto AECID "Desarrollo de un programa de capacitación científico técnica y cre- ación de una estación experimental para el estudio del trópico seco" D/7592/07 y parcialmente económicamente subvencionado por el Programa Horticultura Ambiental del IRTA.

\section{REFERENCIAS}

Aguayo, A., Pauchard, A., Azócar, G., Parra, O. 2009. Cambio del uso del suelo en el centro sur de Chile a fines del siglo XX. Entendiendo la di- námica espacial y temporal del paisaje. Revista chilena de historia na- tural 82: 361-374.

Chuvieco, E., Riaño, D., Aguado, I., Cocero, D. 2002. Estimation of fuel moisture content International Journal of Remote Sensing 23: 2145- 2162.

Chuvieco, E. 1996. Fundamentos de Teledetección. $3^{\mathrm{a}}$ edición revisada. Ediciones RIALP, Madrid. España

Correa, A. 2000. Evaluación de un paisaje fragmentado para la conserva- ción y recuperación de biodiversidad. Tesis de Maestría. Centro Agronómico Tropical de Investigación y Enseñanza. Turrialba, Costa Rica. 
Congalton, R. 1988. A comparision of sampling schemes used in generating error matrices for assessing the acuarency of maps generated from re- motely sensed data. Photogrametric Enginneringand Remote Sensing 54: 593-600.

Di Rienzo, J., Casanoves, F., Balzarini, M., González, L., Tablada, M., Ro- bledo, C. 2010. InfoStat versión 2010. Grupo InfoStat, FCA, Universidad Nacional de Córdoba, Argentina. Consultado el 10 de febrero 2013. Disponible en http://www. infostat.com.ar.

ERDAS, Inc. 1997. Field Guide. Fifth edition, revised and expanded. Atlanta, Georgia, USA.

Esseen, P., Jansson, K., Nilsson, M. 2006. Forest edge quantification by line intersect sampling in aerial photographs. Forest Ecology and Man- agement 230:32-42.

FAO 1996. Forest resources assessment 1990. Survey of tropical forest cover and study of change processes. Number 130, Roma Italia.

Henao, S. 1988. Introducción al manejo de cuencas hidrográficas. Univer- sidad de Santo Tomas, Centro de Enseñanza Desescolarizada, Edicio- nes Usta, Bogotá, Colombia. 398 p.

INIDE 2005. VIII Censo de Población y IV de vivienda. Instituto Nacional de Información de Desarrollo. Gobierno de Reconciliación y Unidad Nacional. Consultado: 04 Julio 2013. Disponible en http:/www.inide.gob.ni/cen-sos2005/CifrasMun/ EsteliTPDF/Estel\%C3\%AD.pdf.

INAFOR 2008. Inventario Forestal de Nicaragua. Instituto Nacional Forestal, Managua, Nicaragua. $232 \mathrm{pp}$

Krummel, J., Gardner, R., Sugihara, G., O’Neill, R., Coleman, P. 1987. Land- scape patterns in a disturbed environment. Oikos 48:321-324.

Laurence, W., Yensen, E. 1991. Predicting the impacts of edge effects in fragmented habitats. Biological Conservation 55: 77-92.

Lambin, E. 1997. Modelling deforestation processes: a review tropical ecosystem environment observations by satellites. TREE Series B., Re- search Report No. 1. European Commission Joint Research Centre- Institute for Remote Sensing Applications-European Space Agency, Luxembourg.

López, R. 2002. Producción y conservación en equilibrio en el área protegía Miraflor Moropotente, Estelí, Nicaragua: sistematización de experiencias. $1^{a}$. Colección: cuadernos de sistematización; No.2 pp. 118. ADESO “Las segovias"/ PROARCA/ CAPAS. Esteli, Nicaragua. ISB: 99924-836-3-6.

Maass, J., Balbanera, P., Castillo, A., Daily, G.C., Mooney, H.A., Ehrlich, P., et al. 2005. Ecosystem services of tropical dry forests: insights from longterm ecological and social research on the Pacific Coast of Mexico. Ecology and Society 10(1): 17

Murcia, C. 1995. Edge effects in fragmented forests: implications for con- servation. Trends in Ecology and Evolution 10: 58-62.

McGarigal, K., Cushman, S., Neel, M., Ene. E. 2002. FRAGSTATS: Spatial Pattern Analysis Program for Categorical Maps. Computer software program produced by the authors at the University of Massachusetts, Amherst, MA. USA.

MARENA et al 2004. Plan De Manejo Del Paisaje Terrestre Protegido Miraflor Moropotente. Ministerio de Ambiente y Recursos Naturales. Managua Ni- caragua. 273 pp. Disponible en: http://www.sinia.net.ni/wamas/documen-tos/ PM/Plan\%20de\%20Manejo\%20Miraflor\%20 Moropotente.pdf

Mendoza, F. 2010. Análisis Multitemporal del Cambio de Uso del Suelo en base a Imágenes Satelitales de los territorios indígenas de Mayangna- Sauni As, MayangnaSauni Bas, Sikilta, Matung Bak / Sauni Arungka, SIPBAA, Layasiksa y el área afectada por el Huracán Félix en 2007 para el período de tiempo 2005 - 2007/08 en los Departamentos de Ji- notega y la RAAN, Nicaragua. Managua, Nicaragua. Disponible en: http://masrenace.wikispaces. com/file/view/Analisis + multitemporal+Cam bio+Uso+Suelo.pdf 
Nicaragua 1999. Reglamentos de áreas protegidas de Nicaragua (RAPN). Decreto No. 14-99, aprobado el 15 de febrero de 1999. MARENA. La Gaceta - Diario oficial, no 42, del 02 de marzo de 1999. pp. 984-993 y no 43, del 03 de marzo de 1999, PP. 1008-1015. Disponible en: http://faolex.fao.org/ docs/pdf/nic15789.pdf

Nicaragua 2010. Uso Potencial de la Tierra, compendio de mapas. 1a ed. Ministerio Agropecuario y Forestal, (MAGFOR). Managua, Nicaragua. 148 p. ISBN : 978-99924-992-1-4.

Ojima, D., Galvin, K., Turner, B.1994. The Global lmpact of Land-use Change. BioScience 44 (5):300-304.

PANIF 1999. Agricultura de Laderas, Áreas Protegidas y Agroturismo: Una Alternativa para aumentar los ingresos de los productores en una Zona con belleza escénica. Estelí, Nicaragua.

Patton, D. 1975. A diversity index for quantifying habitat edge. Wildlife So- ciety Bulletin 394: 171173.

Rau, J., Gantz, A. 2001. Fragmentación del bosque nativo del sur de Chile: efectos del área y la forma sobre la biodiversidad de aves. Boletín de la Sociedad de Biología de Concepción 72: 109-119.

Rau, J., Gantz, A., Torres, G. 2000. Estudio de la forma de fragmentos bos- cosos sobre la riqueza de especies de aves al interior y exterior de áreas silvestres protegidas. Gestión Ambiental 6: 33-40.

Ravera, F. 2007. Degradación y gestión sostenible de los recursos: actores, conflictos y horizontes de diálogo. Una investigación participativa en un área protegida del trópico seco Nicaragüense. Tesis doctoral. Bellaterra, España. 135 pp.
Skole, D., Chomentowski, W., Salas, W., Nobre A. 1994. Physical and Human Dimensions of Deforestation in Amazonia. Bioscience 44 (5):314322.

Stevens, W. 2001. Introducción de vegetación. En: W. Stevens, C. Ulloa, A. Pool, O. Montiel, O. (eds.). Flora de Nicaragua, pp. 1-23. Mono- graphs in Systematic Botany from the Missouri Botanical Garden 85: i-xlii, 1-2666.

Ticona, W. 2011. Estudio de cambio de uso de suelo y escenarios de cambio climático en los municipios de Somoto y Macuelizo. En: presentación en la 3 a. Feria Mesoamericana de postgrados Mexicanos de calidad, 29-30 septiembre 2011, Universidad Nacional de Ingeniería, Managua, Nicaragua. CONACYT y CONICYT. Disponible en: http:// www.conacyt.gob.mx/Becas/feria/Documents/3aUso_suelo_mu-nicipios_Somoto_Macuelizo.pdf

Usher, M.B. 1987. Effects of fragmentation on communities and populations: a review with applications to wildlife conservation. En: Saunders, D.A.,

G.W. Arnold, A.A. Burbidge, Hopkins, A.J.M. (eds). Nature conservation: the role of remmants of native vegetation, pp 103-121. Surrey Beatty and Sons, Chiping Norton, Australia.

Velázquez, A., Mas, J., Palacio, J., Díaz, R., Mayorga, C., Alcantara, R., Fer- nandez, T. 2007. Análisis de cambio de uso del suelo. Informe técnico convenio INE-Instituto de Geografía, UNAM., Ciudad de México DF, México.

Williamson, M. 2006. Análisis multitemporal para la detección de cambios en el uso del suelo en tres municipios afectados por el huracán Juana. CISAURACCAN. Costa Caribe. Nicaragua. 